\title{
Intelligent Joint Admission Control for Next Generation Wireless Networks
}

\author{
Mohammed M. Alkhawlani \\ Faculty of Science and Engineering \\ University of Science and Technology \\ Sanaa, Yemen
}

\author{
Fadhl M. Al-Akwaa \\ Biomedical Engineering Department \\ University of Science and Technology \\ Sanaa, Yemen
}

\author{
Abdulqader M. Mohsen \\ Computer Science Department \\ University of Science and Technology \\ Sanaa, Yemen
}

\begin{abstract}
The Heterogeneous Wireless Network (HWN) integrates different wireless networks into one common network. The integrated networks often overlap coverage in the same wireless service areas, leading to the availability of a great variety of innovative services based on user demands in a costefficient manner. Joint Admission Control (JAC) handles all new or handoff service requests in the HWN. It checks whether the incoming service request to the selected Radio Access Network (RAN) by the initial access network selection or the vertical handover module can be admitted and allocated the suitable resources. In this paper, a decision support system is developed to address the JAC problem in the modern HWN networks. This system combines fuzzy logic and the PROMETHEE II multiple criteria decision making system algorithm, to the problem of JAC. This combination decreases the influence of the dissimilar, imprecise, and contradictory measurements for the JAC criteria coming from different sources. A performance analysis is done and the results are compared with traditional algorithms for JAC. These results demonstrate a significant improvement with our developed algorithm.
\end{abstract}

Keywords- Heterogeneous Wireless Network; Radio Access Network; PROMETHEE; Joint Admission Control

\section{INTRODUCTION}

Heterogeneous Wireless Network (HWN) is defined as a new type of wireless networks where anyone can communicate with anyone else, anywhere and anytime, or enjoy any service of any network operator, through any network of any service provider in the most efficient and optimal way according to the user criteria. The current Radio Resource Management (RRM) solutions and mechanisms for the wireless networks consider only the case of a single Radio Access Technology (RAT) where mobile users can only access that RAT and co-existed sub-networks can only be operated independently. The needs for supporting various applications and services and for providing ubiquitous coverage in the HWN require more complex and intelligent RRM techniques that enable the coordination among the different RATs.

Joint Admission Control (JAC) handles all new or handoff service requests in the HWN. It checks whether the incoming service request to the selected RAT by the initial access network selection algorithm or the vertical handover algorithm selection can be admitted. Then, it allocates the required resources and guarantees the QoS constraints for the service. The relationship between JAC and local admission controls of the involved RATs is highly dependent on the level of coupling and type of relationship between the Common RRM (CRRM) entity and RRM entities of the coupled networks [2].

The most important related work of the JAC problem is presented in the next section. A brief overview for PROMETHEE and FLC is presented in section III. The proposed JAC algorithm for HWN environments is presented in Section IV. The simulation models and performance metrics are presented in section V. The performance evaluation of the proposed algorithm is carried out in Section VI. The conclusions and future works are presented in Section VII.

\section{RELATED WORK}

O. E. Falowo et al. in paper [1] review the recent call admission control algorithms for heterogeneous wireless networks. The benefits and requirements of JAC algorithms are discussed. The authors examine eight different approaches for selecting the most appropriate RAT for incoming calls in HWN and classify the JAC algorithms based on these approaches. The advantages and disadvantages of each approach are discussed. The same authors in [3] propose a JAC algorithm which considers the users preference in making an admission decision and a specific case where the user prefers to be served by the RAT which has the least service cost is modeled and evaluated. In [4] a JAC scheme for multimedia traffic that maximizes the overall network revenue with QoS constraints over coupled WLAN and CDMA cellular network is considered. X. G. Wang et al. [5] propose an adaptive call admission control for integrated cellular and WLAN network. In the proposed scheme, call admission decisions are based on requested QoS and availability of radio resources in the considered RATs. D. Karabudak et al. [6] propose a call admission control scheme for the heterogeneous network using genetic algorithm. The objectives of the scheme are to achieve maximum wireless network utilization and meet QoS requirements. A network capacity policy based joint admission controller is presented by K. Murray et al. [7], [8]. D. Qiang et al. in [9] propose a joint admission control scheme for multimedia traffic that exploits vertical handoffs as an effective tool to enhance radio resource management while guaranteeing handoff users QoS requirements. The network resources utilized by the vertical handoff user are captured by a link utility function. X. Li et al. in [10] propose an efficient joint session admission control scheme that maximizes overall network revenue with QoS constraints over both the WLAN and the TD-SCDMA cellular networks. 
In paper [11], the authors propose a call admission control reservation algorithm that takes resource fluctuations into consideration. They consider two types of applications denoted by wide-band and narrow band. The performance of the algorithm is modeled through a queuing theory approach and its main performance measures are compared with a conventional algorithm through simulation. The authors in paper [12], propose an algorithm, which incorporates traditional Admission Control (AC) and Wiener Process (WP)based prediction algorithms to determine when to carry out access service network gateway relocation. The authors further develop an analytical model to analyze the proposed algorithm. Simulations are also conducted to evaluate the performance of the proposed algorithm.

The main contribution of this paper is the development of a new class of JAC algorithms that are based on hybrid parallel Fuzzy Logic (FL) based decision and PROMETHEE II (Preference Ranking Organization Method for Enrichment Evaluation II) MCDM systems. This class of algorithms represents the first attempt to develop adaptive, flexible, and scalable JAC algorithms that are utilizing the advantages of hybrid parallel FL decision making systems and PROMETHEE II method. FL helps out in reducing the complexity involved on the JAC decision in several ways. First, the data, information, and measurements that have to be taken into account in the JAC are in general very dissimilar, imprecise, contradictory, and coming from different sources. As a result of that, a FL based solution has been thought to be a good candidate for reaching suitable JAC decisions from such imprecise and dissimilar information. Second, JAC solution has to be able to response to the changing conditions of the heterogeneous environments and the accumulated experience of the operators and users. FL based solution is easy to modify by tuning and adjusting the inference rules and membership functions. The application of parallel FL rather than traditional FL achieves more advantages for the JAC solution. The idea of the parallel Fuzzy Logic Control (FLC) reduces the number and complexity of the inference rules used in the FL based solution, which helps out in achieving more scalable solutions. In a very complex and uncertain decision environments, MCDM can sufficiently reduce the uncertainty and doubt about the alternatives and allows a reasonable choice to be made from among them.

\section{PROMETHEE AND FLC}

The PROMETHEE (Preference Ranking Organization Method for Enrichment Evaluation) method was developed by Brans and Vincke in 1985 [13]. The PROMETHEE I method can provide the partial ordering of the decision alternatives, whereas, PROMETHEE II method can derive the full ranking of the alternatives. In this method, pair-wise comparison of the alternatives is performed to compute a preference function for each criterion. Based on this preference function, a preference index for alternative $i$ over alternative $i$ ' is determined. This preference index is the measure to support the hypothesis that alternative $\mathrm{i}$ is preferred to alternative $\mathrm{i}$ '. The PROMETHEE method can classify the alternatives which are difficult to be compared because of a trade-off relation of evaluation standards as non-comparable alternatives. It is quite different from Analytic Hierarchy Process (AHP) method in that there is no need to perform a pair-wise comparison again when comparative alternatives are added or deleted.

Fuzzy Logic (FL) is a problem solving method based on the theory of fuzzy sets, where variables can have different degrees of membership in different sets. Fuzzy Logic Control (FLC) is based on the principles of FL. FLC is a non-linear control method, which attempts to apply the expert knowledge of an experienced user to the design of a controller. Any FLC system contains three stages, the input stage, the processing stage and the output stage. The input stage maps the real valued numbers into fuzzy sets and defines their membership functions. The processing stage maps the input fuzzy sets into output fuzzy sets by combining a set of IF-THEN rules that represents the human knowledge about the problem. The output stage maps the output fuzzy sets into real valued numbers. Mamdani style fuzzy inference system has been used in our work. The idea behind using a Mamdani style is that the rules of the system can be easily described by the humans in terms of fuzzy variables. Thus we can effectively model a complex non-linear system with common sense rules on fuzzy variables [15].

\section{JAC SOLUTION}

A novel JAC algorithm is developed in this section. The algorithm has two main components, the FL based control component and the MCDM component. The input criteria values of the MCDM are the outputs of the FL based control subsystems in the first component. The criteria with more importance to the operator and user can be assigned higher weight. Our algorithm considers five different decision criteria. It consider the Received Signal Strength (RSS), the Signal-toNoise Ratio (SNR), the Resources Availability (RA), the Service Type (ST), and the Mobile Station Speed (MSS) criteria.

\section{A. FLC Component}

Our JAC algorithm contains five FL based subsystems. Each subsystem considers one of the JAC criteria mentioned above. Every subsystem has $\mathrm{x}$ output variables, where $\mathrm{x}$ is the number of existing RATs. Every output variable describes the probability of acceptance for the admission request in one of the existing RATs. Figure 1 shows a sample for an output variable with its membership functions.

For simplicity, only ST Subsystem is considered as an example. The Figures 2 and 3 show the membership functions of the DelayReqc and RateReqc input variables. In case of three RATs, the output variables will be $S T c 1, S T_{c 2}$, and $S T_{c 3}$

The subsystem has nine rules as shown in Table I.

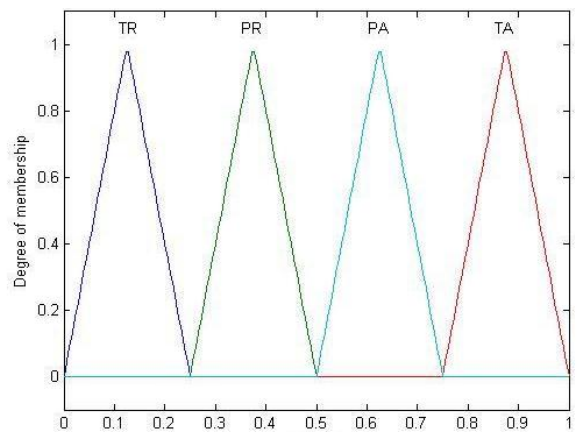

Fig. 1. The output variable 


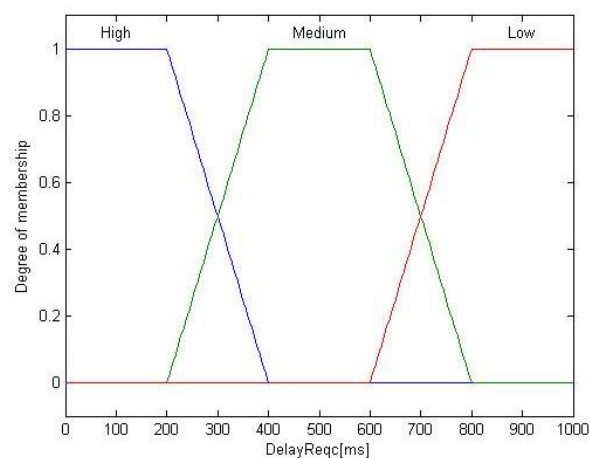

Fig. 2. The input variable DelayReqc

TABLE I

THE INFERENCE RULES OF THE ST FUZZY BASED SYSTEM

\begin{tabular}{cccccc} 
Rule No. & DelayReqc & RateReqc & STc1 & STc2 & STc3 \\
\hline 1 & H & L & TA & TR & PR \\
2 & H & M & PA & PR & PA \\
3 & H & H & PA & PA & PA \\
4 & M & L & PA & PR & PA \\
5 & M & M & PA & PA & PA \\
6 & M & H & PR & PA & PA \\
7 & L & L & PA & PA & PA \\
8 & L & M & TR & TA & TA \\
9 & L & H & TR & TA & PA \\
\hline
\end{tabular}

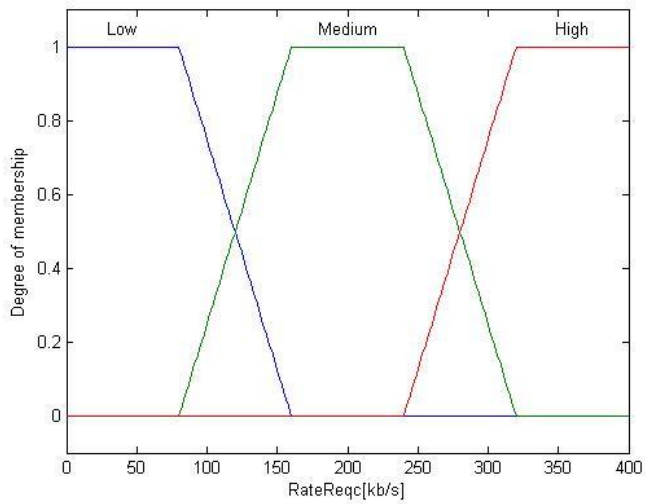

Fig. 3. The input variable RateReqc

\section{B. MCDM Component}

The MCDM system takes the outputs of the FL subsystems as its input and calculates the total ranking value for all alternatives. The procedural steps as involved in PROMETHEE II method can be summarized as follows [14]:

Step 1: The decision matrix for the different alternatives against the set of criteria can be written as shown in equation 1.

$$
N D M=\left(\begin{array}{ccc}
R S S_{c 1} & R S S_{c 2} & R S S_{c 3} \\
S N R_{c 1} & S N R_{c 2} & S N R_{c 3} \\
R A_{c 1} & R A_{c 2} & R A_{c 3} \\
M S S_{c 1} & M S S_{c 2} & M S S_{c 3} \\
S T_{c 1} & S T_{c 2} & S T_{c 3}
\end{array}\right)
$$

Step 2: Normalize the decision matrix using a suitable normalization method. In our approach, since all the outputs of FL subsystems are in the range $[0,1]$, there is not any need to scale and normalize the criteria performance against alternatives.

Step 3: the evaluative differences of ith alternative with respect to other alternatives are calculated. This step involves the calculation of differences in criteria values between different alternatives pair-wise.

Step 4: the preference function $P_{j}\left(i, i^{\prime}\right)$ is calculated. Many types of generalized preference functions are proposed so far. In our algorithm, the following simplified preference function is adopted.

$$
P_{j}\left(i, i^{\prime}\right)=\left\{\begin{aligned}
0 & \text { if } R_{i j} \leq R_{i^{\prime} j} \\
\left(R_{i j}-R_{i^{\prime} j}\right) & \text { if } R_{i j}>R_{i^{\prime} j}
\end{aligned}\right.
$$

Step 5: the next step is to decide on the relative importance of each of the attributes involved in the decision about admission control. For this purpose, each of the attributes is assigned a specific weight, such that

$$
T W=W_{r s s}+W_{s n r}+W_{r a}+W_{m s s}+W_{s t}=1
$$

where $W_{r s s}$ is the assigned weight for the received signal strength criterion. $W_{r a}$ is the assigned weight for the resource availability criterion. $W_{s n r}$ is the assigned weight for the signal to noise ratio criterion. $W_{m s s}$ is the assigned weight for the mobile station speed criterion. $W_{s t}$ is the assigned weight for the service type criterion. $T W$ is the total weight and is calculated using 3 .

Step 6: the aggregated preference function is calculated as follows

$$
\operatorname{APF}\left(i, i^{\prime}\right)=\frac{\sum_{j=1}^{m} W_{j} P_{j}\left(i, i^{\prime}\right)}{\sum_{j=1}^{m} W_{j}}
$$

where $W_{j}$ is the relative weight of $j t h$ criterion.

Step 7: Determine the Leaving Outranking Flow (LOF) and Entering Outranking Flow (EOF) as follows:

$$
\begin{array}{r}
L O F_{i}=\frac{1}{1-n} \sum_{i^{\prime}=1}^{n} \operatorname{APF}\left(i, i^{\prime}\right) \\
E O F_{i}=\frac{1}{1-n} \sum_{i^{\prime}=1}^{n} A P F\left(i^{\prime}, i\right)
\end{array}
$$

where $n$ is the number of alternatives. The leaving (positive) flow expresses how much an alternative dominates the other alternatives, while the entering (negative) flow denotes how much an alternative is dominated by the other alternatives.

Step 8: the Net Outranking Flow (NOF)for each alternative

$$
N O F_{i}=L O F_{i}-E O F_{i}
$$

The higher value of NOF, the better is the alternative. Thus, the best alternative is the one having the highest NOF value.

\section{THE PERFormance Evaluation}

Our proposed solution is evaluated using the simulation approach. This section presents the used performance metrics 
and simulation models.

\section{A. The performance metrics}

The performance of the proposed JAC algorithm is evaluated using three performance evaluation metrics. The used metrics can be described briefly as follows.

- Blocking probability $\left(P_{b}\right)$ is defined as the ratio of the number of blocked users to the total number of new users requesting admission. A user is blocked if at the session start the JAC algorithm assigns a bit rate of 0 $\mathrm{kb} / \mathrm{s}$.

- $\quad$ Outage probability $\left(P_{o}\right)$ is calculated as the ratio of the number of users not fulfilling their Guaranteed Bit Rate (GBR) requirement, to the total number of admitted users.

- Unsatisfied user probability $\left(P_{u}\right)$ that could be calculated based on $P_{b}$ and Po as shown in equation 8

$$
P_{u}=1-\left(1-P_{b}\right)\left(1-P_{o}\right)
$$

\section{B. The simulation environment}

A modified version of MATLAB based simulator called RUNE [16] has been used. Our models developed in [17] have been updated to be used in this work. The simulation environment defines a system model, a mobility model, a propagation model, and services model. The system model specifies the type of networks and the number and characteristics of the cells. Our system model considers the coexistence of six types of RATs. The characteristics of the RATs are summarized in Table II.

TABLE II.

SYSTEM MODELS DETAILS

\begin{tabular}{|c|c|c|c|c|}
\hline $\begin{array}{c}\text { RAT } \\
\text { No. }\end{array}$ & $\begin{array}{c}\text { Multiple } \\
\text { Access Type }\end{array}$ & $\begin{array}{c}\text { Antenna } \\
\text { Type }\end{array}$ & $\begin{array}{c}\text { Cell } \\
\text { Radius }\end{array}$ & $\begin{array}{c}\text { Number } \\
\text { of Cells }\end{array}$ \\
\hline RAT1 & CDMA/WWAN & Omni-directional & $750 \mathrm{~m}$ & 7 \\
\hline RAT2 & CDMA/WMAN & Omni-directional & $375 \mathrm{~m}$ & 12 \\
\hline RAT3 & CDMA/WLAN & Omni-directional & $100 \mathrm{~m}$ & 27 \\
\hline
\end{tabular}

The mobility model simulates the mobility of the users in the system environment. In our mobility model, the mobiles are randomly distributed over the system. In every slot each mobile is moved a random distance in a random direction at defined time steps.

The propagation model simulates the different losses and gains during the signal propagation between the transmitter and the receiver in the system environment. The wireless propagation model used in this paper is described in a logarithmic scale as in equation 9.

$$
G=G_{D}+G_{F}+G_{R}+G_{A}
$$

Equation 9 contains four components, the first component is the distance attenuation $G D$ that is calculated by Okumura-Hata formula. The second component is the shadow fading $G F$ that is modeled as a log-normal distribution with standard deviation of $6 \mathrm{~dB}$ and $0 \mathrm{~dB}$ mean. The third component is the Rayleigh fading $G R$ that is modeled using a Rayleigh distribution. The forth component is the antenna gain
$G A$ that adds the antenna gain in $\mathrm{dB}$.

The services model specifies the type of services and their percentages of use in the system environment. The ith service is mainly characterized by its bit rate requirement RateReqc and delay requirement DelayReqc. The users are generated according to Poisson process. The service holding time is exponential distribution with mean holding time equals to 150 seconds.

\section{THE RESULTS STUDY}

Three different alternative algorithms are simulated and evaluated against our proposed solution. The first alternative does not take into account the JAC concept (It is denoted as NJAC) and the local RRM entities take the full responsibility to admit or reject the users. The second reference algorithm is denoted as Load-based JAC (LJAC) and it selects the least loaded RAT for new or handoff request. Finally, the third algorithm selects the RAT in which the mobile measures the strongest received signal strength, and it is denoted as Signal Strength JAC (SSJAC). In all the three cases, once the RAT has been selected, the bandwidth assigned to each user is the maximum bandwidth considered for this RAT for this type of service. Some simulation results for different sets of users are presented in this section.

Table III and Figure 4 illustrate some numerical results for the $P_{b}$ values in all algorithms. The results show that our solution achieve good performance enhancement over all algorithms. On average, our algorithm achieves around 18\%, $7 \%$, and $12 \%$ enhancement over NJAC, LJAC, and SSJAC algorithms respectively. Better results can be gained if more suitable weights are used.

Table IV and Figure 5 illustrate some numerical results for the $P_{o}$ values in all algorithms. The results show that our solution achieve good performance enhancement over all algorithms. On average, our algorithm achieves around 15\%, $4 \%$, and $9 \%$ enhancement over NJAC, LJAC, and SSJAC algorithms respectively. Better results can be gained if more suitable weights are used.

Table V and Figure 6 illustrate some numerical results for the $P_{u}$ values in all algorithms. The results show that our solution achieve good performance enhancement over all algorithms. On average, our algorithm achieves around $25 \%$, $9 \%$, and $17 \%$ enhancement over NJAC, LJAC, and SSJAC algorithms respectively. Better results can be gained if more suitable weights are used.

TABLE III

$P_{b}$ VALUES IN ALL ALGORITHMS

\begin{tabular}{ccccc}
\hline $\begin{array}{c}\text { No. of } \\
\text { Users }\end{array}$ & $\begin{array}{c}\text { Our } \\
\text { solution }\end{array}$ & $\begin{array}{c}\text { NJAC } \\
\text { solution }\end{array}$ & $\begin{array}{c}\text { LJAC } \\
\text { solution }\end{array}$ & $\begin{array}{c}\text { SSJAC } \\
\text { solution }\end{array}$ \\
\hline 50 & 0.01 & 0.09 & 0.04 & 0.07 \\
150 & 0.04 & 0.18 & 0.09 & 0.14 \\
250 & 0.09 & 0.29 & 0.18 & 0.22 \\
350 & 0.14 & 0.37 & 0.24 & 0.3 \\
450 & 0.19 & 0.48 & 0.31 & 0.41 \\
\hline
\end{tabular}




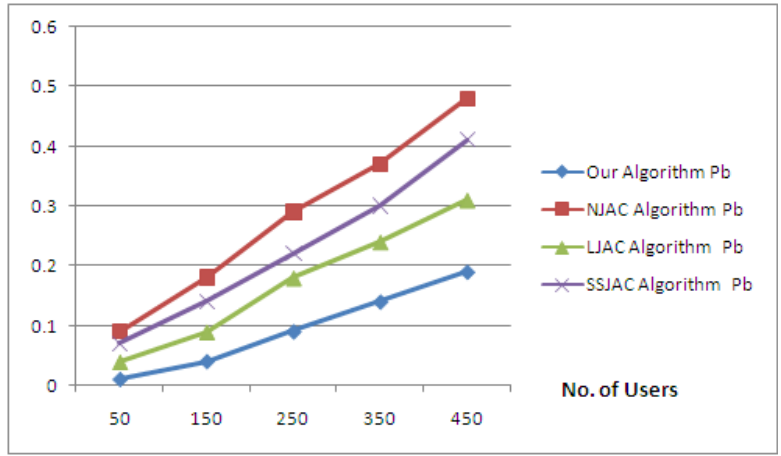

Fig. 4. $\quad P_{b}$ values for all algorithms

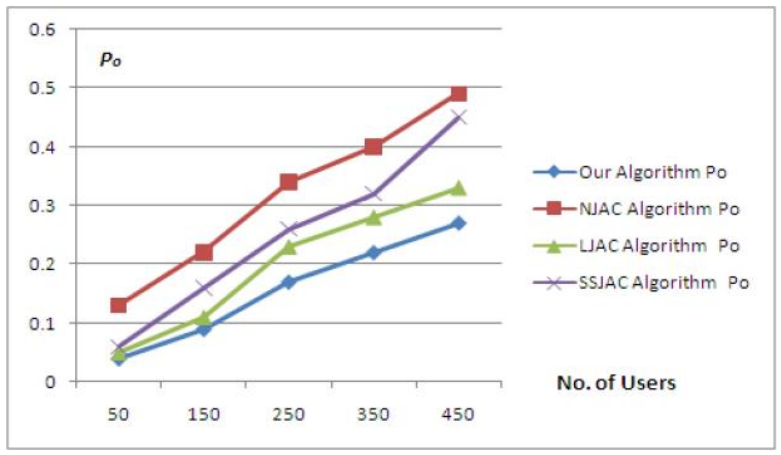

Fig. 5. Po values for all algorithms

TABLE IV

$P_{o}$ VALUES IN ALL ALGORITHMS

\begin{tabular}{ccccc}
\hline $\begin{array}{c}\text { No. of } \\
\text { Users }\end{array}$ & $\begin{array}{c}\text { Our } \\
\text { solution }\end{array}$ & $\begin{array}{c}\text { NJAC } \\
\text { solution }\end{array}$ & $\begin{array}{c}\text { LJAC } \\
\text { solution }\end{array}$ & $\begin{array}{c}\text { SSJAC } \\
\text { solution }\end{array}$ \\
\hline 50 & 0.04 & 0.13 & 0.05 & 0.06 \\
150 & 0.09 & 0.22 & 0.11 & 0.16 \\
250 & 0.17 & 0.34 & 0.23 & 0.26 \\
350 & 0.22 & 0.4 & 0.28 & 0.32 \\
450 & 0.27 & 0.49 & 0.33 & 0.45 \\
\hline
\end{tabular}

TABLE V

$P_{U}$ VALUES IN ALL ALGORITHMS

\begin{tabular}{ccccc}
\hline $\begin{array}{c}\text { No. of } \\
\text { Users }\end{array}$ & $\begin{array}{c}\text { Our } \\
\text { solution }\end{array}$ & $\begin{array}{c}\text { NJAC } \\
\text { solution }\end{array}$ & $\begin{array}{c}\text { LJAC } \\
\text { solution }\end{array}$ & $\begin{array}{c}\text { SSJAC } \\
\text { solution }\end{array}$ \\
\hline 50 & 0.0496 & 0.2083 & 0.088 & 0.1258 \\
150 & 0.1264 & 0.3604 & 0.1901 & 0.2776 \\
250 & 0.2447 & 0.5314 & 0.3686 & 0.4228 \\
350 & 0.3292 & 0.622 & 0.4528 & 0.524 \\
450 & 0.4087 & 0.7348 & 0.5377 & 0.6755 \\
\hline
\end{tabular}

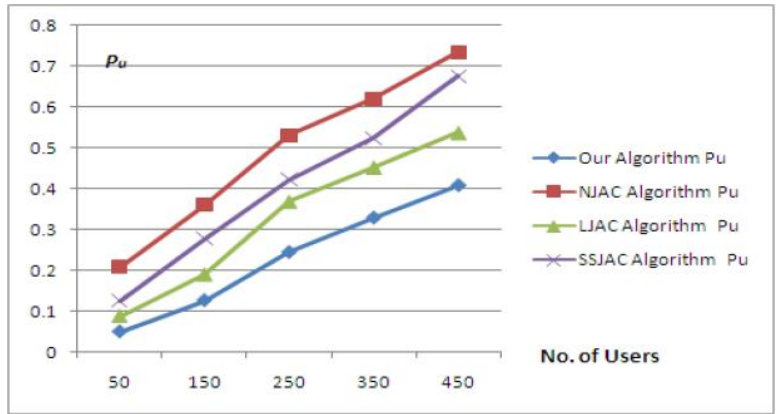

Fig. 6. $\quad P_{u}$ values for all algorithms

In one hand and for reasons of simplicity and computational complexity, the simulation cannot be carried out at higher number of users. In the other hand, the achieved simulation results show that our algorithm outperforms the reference algorithms, and a clear monotonic increasing relationship could be directly observed between the number of users and the performance metrics. To check if there is any linear relationship between the number of users and the achieved performance metrics, the Pearsons Correlation Coefficient (PCC) is used. PCC investigates the strength and direction of a linear relationship between two random variables. $\mathrm{PCC}=+1$ means very strong positive linear relationship. $\mathrm{PCC}=-1$ means very strong negative linear relationship. $\mathrm{PCC}=0$ means no linear relationship is existed between both variables. The results shows that the values of the PCC are all around +1 which means very strong positive linear relationship and we hence expect that our algorithm will keep outperforming the other algorithms at very high number of users.

\section{CONCLUSIONS AND FUTURE WORK}

A suitable design for the JAC algorithms is a highly important issue to achieve the aims of the HWN. This paper explores the issue of JAC in the HWN. A novel JAC algorithm has been designed, implemented, simulated and evaluated. The developed JAC solution attempts to increase the user satisfaction, and decrease the blocking and outage probability.

Our future works can be extended in several directions. An optimum values for the weights of the different criteria can be found using a global optimization method. The developed JAC algorithm can be integrated with other CRRM mechanisms such as Access Network selection (ANS), Joint Congestion Control (JCC), and Vertical Handover (VHO). A joint optimization of these mechanisms can enhance overall system performance. In addition, this study has developed generic JAC algorithms. The algorithms can be tailored to specific wireless standards such as UMTS, IEEE802.16, and IEEE802.11.

\section{REFERENCES}

[1] O. E. Falowo and H. A. Chan, "Joint call admission control algorithms: Requirements, approaches, and design considerations", Computer Communications, vol. 31, no. 6, pp. 1200-1217

[2] J. Prez-Romero, O. Sallent, and R. Agust, "On evaluating beyond 3G radio access networks: architectures, approaches and tools," IEEE 61st Vehicular Technology Conference (VTC 2005-Spring), vol. 5, pp. 2964 - 2968, June 2005

[3] O. E. Falowo, H. A. Chan, "Joint call admission control For next generation wireless network," IEEE Conference on Electrical and Computer Engineering, pp.1151 - 1154, May 2006

[4] Yu. Fei and V. Krishnamurthy, "Optimal joint session admission control in integrated WLAN and CDMA cellular networks with vertical handoff," IEEE Transactions on Mobile Computing, vol. 6, no. 1, pp. 126 - 139, Jan. 2007

[5] X. G. Wang, G. Min, and J. E. Mellor Adaptive QoS control in cellular and WLAN interworking networks, Second International Working Conference on the Performance Modeling and Evaluation of Heterogeneous Networks (HET-NETs '04), West Yorkshire, U.K., July 2004

[6] D. Karabudak, C. Hung, and B. Bing, A call admission control scheme using genetic algorithms, Symposium on Applied Computing (SAC 04), pp.1151 1158, Cyprus, March 2004

[7] K. Murray and D. Pesch, "Policy based access management and handover control in heterogeneous wireless networks," The IEEE Vehicular Technology Conference, vol.5, pp.3319- 3323, Sep. 2004

[8] K. Murray and D. Pesch,"Intelligent network access and inter-system 
handover control in heterogeneous wireless networks for smart space environments," 1st International Symposium on Wireless Communication Systems, pp. 66-70, 2004

[9] D. Qiang, H. Bo, S. Yan, and C. Shan-zhi, "Joint admission control through vertical handoffs in heterogeneous wireless network," Global Mobile Congress (GMC), 2010, pp. 1-5, Nov. 2010

[10] X. Li, H. Ji, P. Si, and L. Zhang, "Joint session admission control scheme in integrated WLAN and 3G networks," 5th International ICST Conference on Communications and Networking in China (CHINACOM 2010), pp.1-5, Aug. 2010

[11] M. Khabazian, O. Kubbar, H. Hassanein, "Call Admission Control with Resource Reservation for Multi-service OFDM Networks,” 2012 International Conference on Computing, Networking and Communications (ICNC), pp.781-785, Feb. 2012

[12] Zong-Hua Liu, Jyh-Cheng Chen,"Design and Analysis of the Gateway Relocation and Admission Control Algorithm in Mobile WiMAX Networks," IEEE Transactions on Mobile Computing, pp.5-18, Jan. 2012
[13] M. Doumpos, and C. Zopounidis, "A multi-criteria classification approach based on pair-wise comparison," European Journal of Operational Research, pp. 378-389, 2004

[14] V. Manikrao Athawale, S. Chakraborty, "Facility location selection using PROMETHEE II method," The 2010 International Conference on Industrial Engineering and Operations Management, pp. 18-22, Jan. 2010

[15] J.S.R. Jang, C.T. Sun, E. Mizutani, Neuro-Fuzzy and soft Computing, Prentice Hall, 1997.

[16] J. Zander and S. Kim, Radio Resource Management for Wireless Networks, Artech House, 2001

[17] Mohammed M. Alkhawlani, "Intelligent Vertical Handover for Heterogonous Networks Using FL And ELECTRE," International Journal of Computer Networks and Communications (IJCNC), Vol.3, No.4, July 2011 